\title{
Preclinical Trial of Noncontact Anthropometric Measurement Using IR-UWB Radar
}

\section{Jinsup Kim}

Hanyang University College of Medicine

Won Hyuk Lee

Hanyang University

\section{Seung Hyun Kim}

Hanyang University College of Medicine

Jae Yoon $\mathrm{Na}$

Hanyang University College of Medicine

Dingyang Wang

Hanyang University

Young-Hyo Lim

Hanyang University College of Medicine

\section{Seok Hyun Cho}

Hanyang University College of Medicine

\section{Sung Ho Cho}

Hanyang University

Hyun-Kyung Park ( $\square$ neopark@hanyang.ac.kr)

Hanyang University College of Medicine

\section{Research Article}

Keywords:

Posted Date: January 19th, 2022

DOI: https://doi.org/10.21203/rs.3.rs-1232519/v1

License: (c) (1) This work is licensed under a Creative Commons Attribution 4.0 International License.

Read Full License 


\title{
Preclinical trial of noncontact anthropometric measurement using IR-UWB radar
}

\author{
Jinsup Kim ${ }^{1,+}$, Won Hyuk Lee ${ }^{2,+}$, Seung Hyun Kim ${ }^{1,+}$, Jae Yoon $\mathrm{Na}^{1}$, Dingyang Wang ${ }^{2}$, \\ Young-Hyo $\mathrm{Lim}^{3}$, Seok Hyun $\mathrm{Cho}^{4}$, Sung Ho $\mathrm{Cho}^{2, *}$, and Hyun-Kyung Park ${ }^{1, *}$
}

\author{
${ }^{1}$ Department of Pediatrics, Hanyang University College of Medicine, Seoul, 04763, Republic of Korea; \\ ${ }^{2}$ Department of Electronics and Computer Engineering, Hanyang University, Seoul, 04763, Republic of Korea; \\ ${ }^{3}$ Division of Cardiology, Department of Internal Medicine, Hanyang University College of Medicine, Seoul, 04763, \\ Republic of Korea; \\ ${ }^{4}$ Department of Otorhinolaryngology, Hanyang University College of Medicine, Seoul, 04763, Republic of Korea; \\ +These authors contributed equally to this work \\ *dragon@hanyang.ac.kr; neopark@hanyang.ac.kr;
}

\begin{abstract}
Anthropometric profiles are important indices for the assessment of medical conditions, including malnutrition, obesity, and growth disorders. Noncontact methods for estimating those parameters could have considerable value in many practical situations, such as the assessment of young, uncooperative infants or children and the prevention of infectious disease transmission. The purpose of this study is to investigate the feasibility of obtaining noncontact anthropometric measurements using the impulse-radio ultrawideband (IR-UWB) radar sensor technique. A total of 45 healthy adults were enrolled, and a convolutional neural network (CNN) algorithm was implemented to analyze data extracted from IR-UWB radar. The differences (root-mean-square error, RMSE) between values from the radar and bioelectrical impedance analysis (BIA) as a reference in the measurement of height, weight, and body mass index (BMI) were 2.78, 5.31, and 2.25, respectively; predicted data from the radar highly agreed with those from the BIA. The intraclass correlation coefficients (ICCs) were $0.93,0.94$, and 0.83 .

In conclusion, IR-UWB radar can provide accurate estimates of anthropometric parameters in a noncontact manner; this study is thus the first supporting the radar sensor as an applicable method in clinical situations.
\end{abstract}

\section{Introduction}

Assessment of anthropometric profiles (height, weight, body mass index [BMI], body circumference, and skin-fold thickness) plays an important role in the evaluation of various medical conditions ${ }^{1,2}$. In particular, a person's height and weight are the core elements of anthropometry and indicators of his or her physical aspects and are crucial in monitoring the state of the disease or assessing growth and development according to nutrition condition in infants and children ${ }^{2,3}$. Changes in weight are important information for monitoring the health of infants and children and evaluating their growth and development.During medical emergencies or serious conditions in the intensive care unit, accurately measuring the weight of patients can be difficult due to difficulty in moving one with mental/physical disabilities; one solution to this type of problem may lie in noncontact measurement ${ }^{4-6}$, which has demonstrated considerable value in the forensic, online business, and virtual environment domains. The recent global COVID-19 pandemic has raised issues related to the transmission of infectious diseases and the urgent need for the development of noncontact technologies.

In general, height and weight are measured indoors using traditional methods involving physical contact, such as a scale. Several studies have been conducted that have employed various techniques to measure these two values using noncontact, accurate, and convenient approaches, including Kinect sensors, 3D structured light sensors, ultrasonic sensors, estimations from images or video, and so on ${ }^{4,7-11}$. Kinect sensors have also accurately measured parameters of the human body with large-scale motion under the assumption that the individual was wearing clothes ${ }^{7}$.However, these methods have not overcome the technical limitations of the sensors thus far ${ }^{7}$.Ultrasonic sensors are cost-effective devices for measuring the size of the human body at distances up to $300 \mathrm{~cm}$ despite variations in body shape ${ }^{9}$. The major difficulty in noncontact weight measurement is characterization of the main anatomical and compartmental components. Spatiotemporal analysis for mining information across the posture variation is needed to measure the body parameters.

Impulse-radio ultrawideband (IR-UWB) radar is a high-precision electromagnetic technique that recognizes the motion of an object at a distance and, unlike video images, lacks privacy issues. This radar has various advantages over other techniques in medical applications, such as its contactless/wireless and license-free nature, ease of application, inexpensiveness, high 
data-processing rate, low exposure risk for the human body, and daily convenience in and out of the hospital. Recently, we introduced a signal model for IR-UWB radar and an algorithm for noncontact, continuous measurement of tiny movements and vital signs (respiratory and heart rates) of the human body, even for small, premature infants ${ }^{6,12-16}$. The results of these studies demonstrated the applicability of IR-UWB radar in the assessment of medical status using distance information. The algorithm for calculating body parameters uses a convolutional neural network (CNN) to analyze patterns extracted from the images from IR-UWB radar. This noncontact sensing technology can measure height and weight without any limitation to the value range and can be implemented with the Internet of Things (IoT) for remote health monitoring in digital healthcare markets, overcoming the issues of contact and infection in the COVID-19 pandemic.

To the best of our knowledge, this work is the first attempt to estimate anthropometric parameters by exploiting the novel, noncontact IR-UWB radar sensor. The purposes of this study are to propose a unique algorithm for measuring these parameters them with radar, to implement a CNN with optimized layers to achieve an algorithm with maximum accuracy, and to evaluate the accuracy of the algorithm and compare it to that of traditional methods.

\section{Materials and Methods}

\section{Subjects}

To acquire IR-UWB radar data, we recruited a total of 45 adults (19 men and 26 women) over the age of 20 from Hanyang University Hospital who participated voluntarily from January to April 2021. This study was approved by the institutional review board of Hanyang University Medical Center (No. 202101-015). All methods were performed in accordance with standard human research ethics guidelines (Declaration of Helsinki) and regulations. Written informed consent was provided by all patients before they were enrolled in the study. Additionally, no participants had a history of mental illness or trauma or diseases of the nervous system or musculoskeletal system.

\section{Experimental Setup and Data Collection}

All experiments for data collection were conducted in the multidisciplinary room of the Inclusive Clinic for Developmental Disorders at Hanyang University Hospital, Seoul, Korea.

\section{Reference Methods}

Height was measured with a calibrated, wall-mounted stadiometer, and bioelectrical impedance analysis (BIA) was conducted using Inbody ${ }^{\circledR} 720$ (Biospace Co., Seoul, Korea; Fig. 1(a)) to measure weight $(\mathrm{kg})$, BMI $\left(\mathrm{kg} / \mathrm{m}^{2}\right)$, muscle mass percentage $(\%)$, skeletal muscle mass percentage (\%), body fat mass percentage (\%), and body water mass percentage (\%). BIA is a useful and well-studied technique for measuring body fat relative to lean mass ${ }^{17}$. Weight was also measured by a digital scale to the nearest $50 \mathrm{~g}$, and height was evaluated to the nearest $0.1 \mathrm{~cm}$.
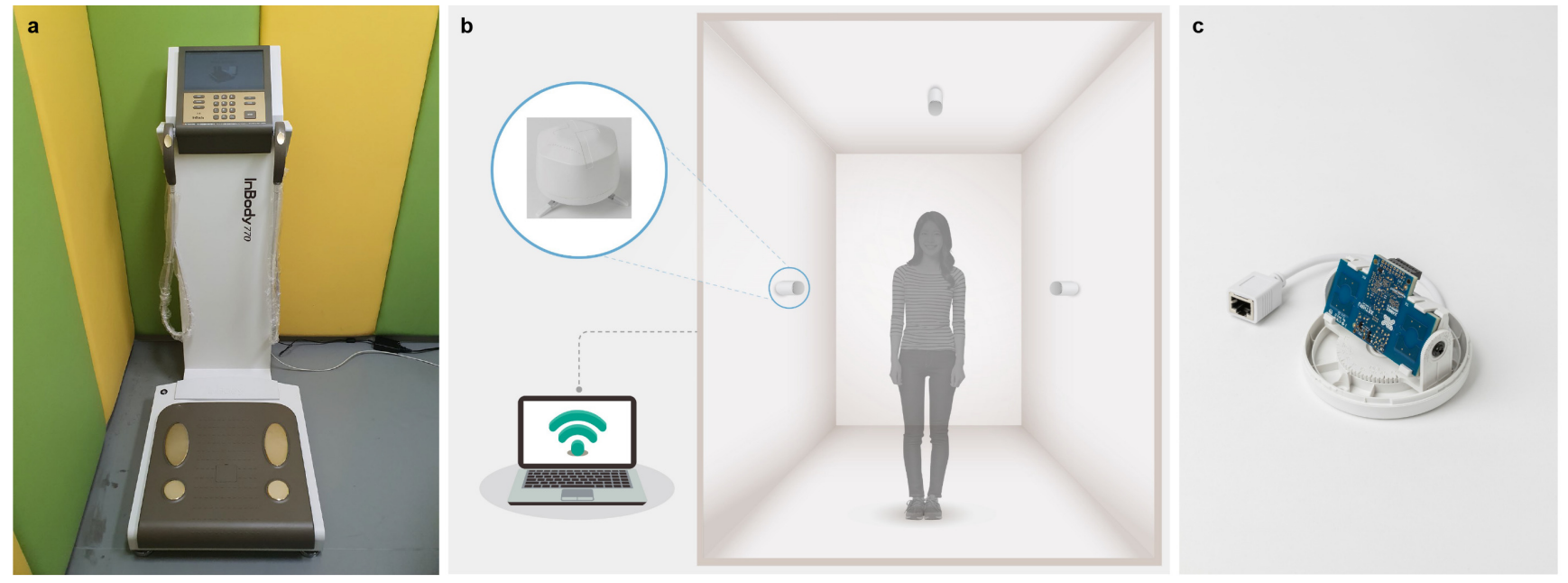

Figure 1. Overall experimental environment for data collection. (a) BIA (Inbody ${ }^{\circledR} 720$ ), (b) radar sensor settings, and (c) IR-UWB radar chip (XK350-120 W0) covered with a plastic cap.

\section{IR-UWB Radar Sensor}

Next, the participant stood motionless for 5 seconds in the middle of the room installed with three commercially available IR-UWB radar sensors (XK350-12 W0, Xandar Kardian, Delaware, USA) for data collection; each radar was encapsulated 
with a white plastic cover (Fig. 1(b)). The room measured $3 \mathrm{~m} \times 3 \mathrm{~m}$ x $2.5 \mathrm{~m}$ wide; one of the three IR-UWB radar sensors was installed in the center of the ceiling of the room, and the other two, on opposite walls 1 meter up from the floor (Fig. 1(c)). The center frequency of the radar sensors used for data collection was $8.748 \mathrm{GHz}$, and the received signal was sampled at 23.328 $\mathrm{GS} / \mathrm{s}$ at the receiver. The bandwidth was approximately $1.5 \mathrm{GHz}$, and the transmitted radiation power was 68.85 microwatts; this transmission power complies with FCC mask indoor standards, and the signals transmitted from each radar sensor did not interfere with each other. The distance resolution was $6.4 \mathrm{~mm}$, which was suitable for capturing minute body movements. The signal transmitted from the radar sensor reached the test participant's body and was reflected back to the receiver, which then transmitted the sampled signals to the PC through USB at a rate of 20 times per second. All radar signals transferred to the PC were processed through MATLAB (MathWorks, MA, USA). All participants were tested 8 times to collect IR-UWB radar data.

\section{Statistical Analysis}

Experimental results data are presented in scatter plots in terms of the root-mean-square error (RMSE), mean absolute error (MAE), intraclass correlation coefficient (ICC) and Pearson correlation coefficient $\left(r^{2}\right)$ values with the reference method due to the characteristics of the data. A scatter plot was used to compare the concordance and correlation between the measured values and the estimated values. Statistical analysis was performed using MATLAB version 2021a.

\section{Algorithm for Measuring Anthropometric Parameters}

\section{System Overview}

The block diagram for the proposed method is shown in Fig. 2. Human body signals collected from three radars are input into the $\mathrm{CNN}$ following preprocessing and image mappingand trained through the CNN together with the measured anthropometric parameters. The dataset for verifying the trained CNN also undergoes same signal processing, and an anthropometric parameter prediction is generated through the trained $\mathrm{CNN}$.

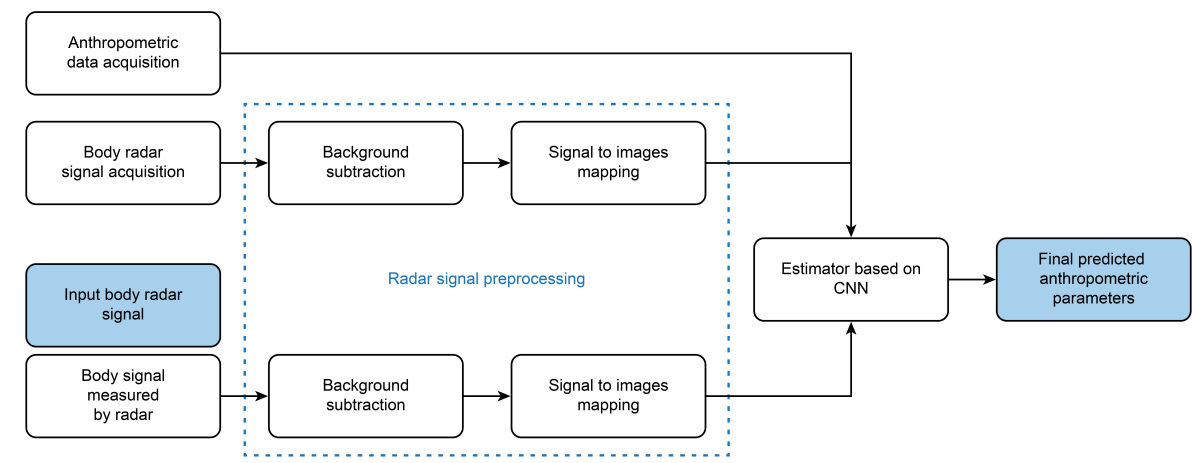

Figure 2. Proposed method for anthropometric parameter estimation using a convolutional neural network.

\section{Radar Signal Preprocessing}

The impulse signal transmitted from the radar sensor is reflected off of not only the participant's body but also objects in the experimental environment. The signal $x_{i, n}[k]$ received from the $i$-th radar sensor at the $n$-th time can be modeled as follows.

$$
x_{i, n}[k]=\sum_{m=1}^{N_{\text {path }}} A_{m, i, n} S\left[k-\tau_{m, i, n}\right]+N[k]
$$

$k$ is a distance index and has a natural number value from 0 to the length of the radar signal. $A$ and $\tau$ are the delay and scaling factors created for the $m$-paths of the transmitted impulse signal returning to the receiver. $N[k]$ is the noise component in the experimental environment ${ }^{18}$. Since the received signal contains not only the signal for the target but also that for the components of the experimental environment, it is not suitable for immediate use in anthropometric parameter measurement; these clutter signals have a larger magnitude than the target signal, which is thus difficult to distinguish. Instead, we remove the clutter signal from the received signal using a background subtraction algorithm, leaving only the target signal, the experimental participants' signals, to be extracted ${ }^{19}$.

In the background subtraction algorithm, the clutter signal $C_{i, n}[k]$ is continuously updated over time, and signal $y_{i, n}[k]$, in which the background signal is removed, is obtained by subtracting the clutter signal $C_{i, n}[k]$ from the original signal $x_{i, n}[k]$. The alpha value can take a value between 0 and 1 ; in this study, it is set to 0.95 . 


$$
\begin{aligned}
y_{i, n}[k] & =x_{i, n}[k]-C_{i, n}[k], \\
C_{i, n}[k] & =\alpha C_{i, n-1}[k]+(1-\alpha) x_{i, n}[k]
\end{aligned}
$$

\section{Height Estimation using Radar Signal Processing}

Height can be obtained through a preprocessed signal extracted only from the radar sensor installed on the ceiling. Since we already know the distance between the radar sensor installed in the ceiling and the ground, if we can accurately measure the distance between the sensor and the top of the head when the participant is standing in the experiment room, we can calculate his or her height. To achieve high measurement accuracy, we used the constant false alarm rate (CFAR) algorithm on the given signal $y_{i, n}[k]$ to measure the height ${ }^{20}$. This algorithm is used to detect a target; one such algorithm is cell-average constant false alarm rate (CA-CFAR), which implements a window of a certain size in one frame of data received from the sensor. First, a threshold is generated according to the CA-CFAR algorithm from signals collected in an experimental environment without a target. A target can then be detected by comparing the generated threshold with the signal received when a target is present. This threshold is created by setting the probability of a false alarm; when there is no target, as many as $N_{c}$ received signals are taken as absolute values after applying the Hilbert transform.

$$
H_{i}[k]=\left[h_{i, 0}[k], h_{i, 1}[k], h_{i, 2}[k], \ldots, h_{i, N_{c}}[k]\right]^{T}
$$

$h_{i, n}[k]$ is the signal resulting from application of the Hilbert transform and absolute value function to the backgroundsubtracted signal $y_{i, n}[k]$ when no one is in the experimental environment,and $H_{i}[k]$ is an array of stacked with up to $N_{c}$ of these signals to determine threshold $T_{i}[k]$. In Eq. (4), the mean value of the signal $H_{i}[k]$ collected in the empty experimental environment is $\sigma_{i}[k], \mu_{i}[k]$ is the standard deviation of $H_{i}[k]$, and $\beta$ is a constant used for adjusting the false alarm rate. The environment-adapted threshold $T_{i}[k]$ is used to obtain the distance from the sensor installed on the ceiling to the tip of the head given signal $y_{i, n}[k]$ collected when a person is present.

$$
T_{i}[k]=\beta \sigma_{i}[k]+\mu_{i}[k]
$$

\section{Proposed CNN Architecture}

Aside from height, the other anthropometric parameters are difficult to obtain through signal processing methods. Most of those measured through BIA are values related to volume or mass, and most of the IR-UWB radar signals are reflected from human skin and cannot penetrate into the human body due to the frequency band used and other characteristics of the transmitted impulse signal ${ }^{21}$. Given these characteristics, the received signal is one-dimensional data whose signal strength is related to distance; it is thus difficult to estimate the indicators related to the human body through a single radar signal. Therefore, we attempt to use all the signals received from the three radars and propose a method for predicting the anthropometric parameters through the maximum likelihood method by imaging the radar signal and applying it to the CNN.

After data preprocessing through the background subtraction algorithm, the training data were generated from radar signals with a size of $100 \times 400$. Since the FPS of the used radar is 20 and the range resolution is $6.4 \mathrm{~mm}$, the radar signal used for training is collected for 5 seconds at distances up to approximately $2.4 \mathrm{~m}$. Then, the data from the 3 radars are stacked and rescaled from 0 to 255 , which matches the 8-bit color depth range. The proposed algorithm uses color images as the input data; therefore, single channel images are converted to RGB channel jpeg images using the conversion function with a given color map. Finally, the image size is resized to $(227,227,3)$, where 3 is the number of RGB color channels. Sample training images are shown in Fig. 3.

The accuracy in estimating an anthropometric parameter depends on the quality of the image generated from the radar signal used as the CNN input. From the preprocessed radar signal, we remove the signal corresponding to the clutter component in the experimental environment and use only the signal corresponding to the target. In this way, features can be extracted through a signal processing method using radar signals without the need for pre-extracted features. Although various types of CNN structures can be applied to radar signals, it is more suitable to predict anthropometric parameters by applying each radar signal to an independent convolution layer rather than attaching all radar signals to one image and using it as input. The datasets created by each radar sensor have characteristics of human signals that depend on the location where it is installed, and since three sensors are used, a late-fusion method, rather than feature-level fusion, is used for the CNN structure, given the multi modality 22,23 .

In our proposed CNN structure, the input image is composed of three preprocessed radar signals. The input images generated from each radar sensor are split, used to train the network, and concatenated in the CAT layer. The size of the filter used in the $2 \mathrm{D}$ convolution layer is $3 \times 3$, and the rectified linear unit (ReLU) layer is used as the activation function. In general, 
the ReLU layer is added after the convolution layer in the CNN structure using images. The operation order of each layer is convolution, ReLU, and average pooling. The final feature is created in the last fully connected layer, and the anthropometric parameters are estimated through a regression layer. Table 1 shows the details of the design of the proposed CNN architecture.

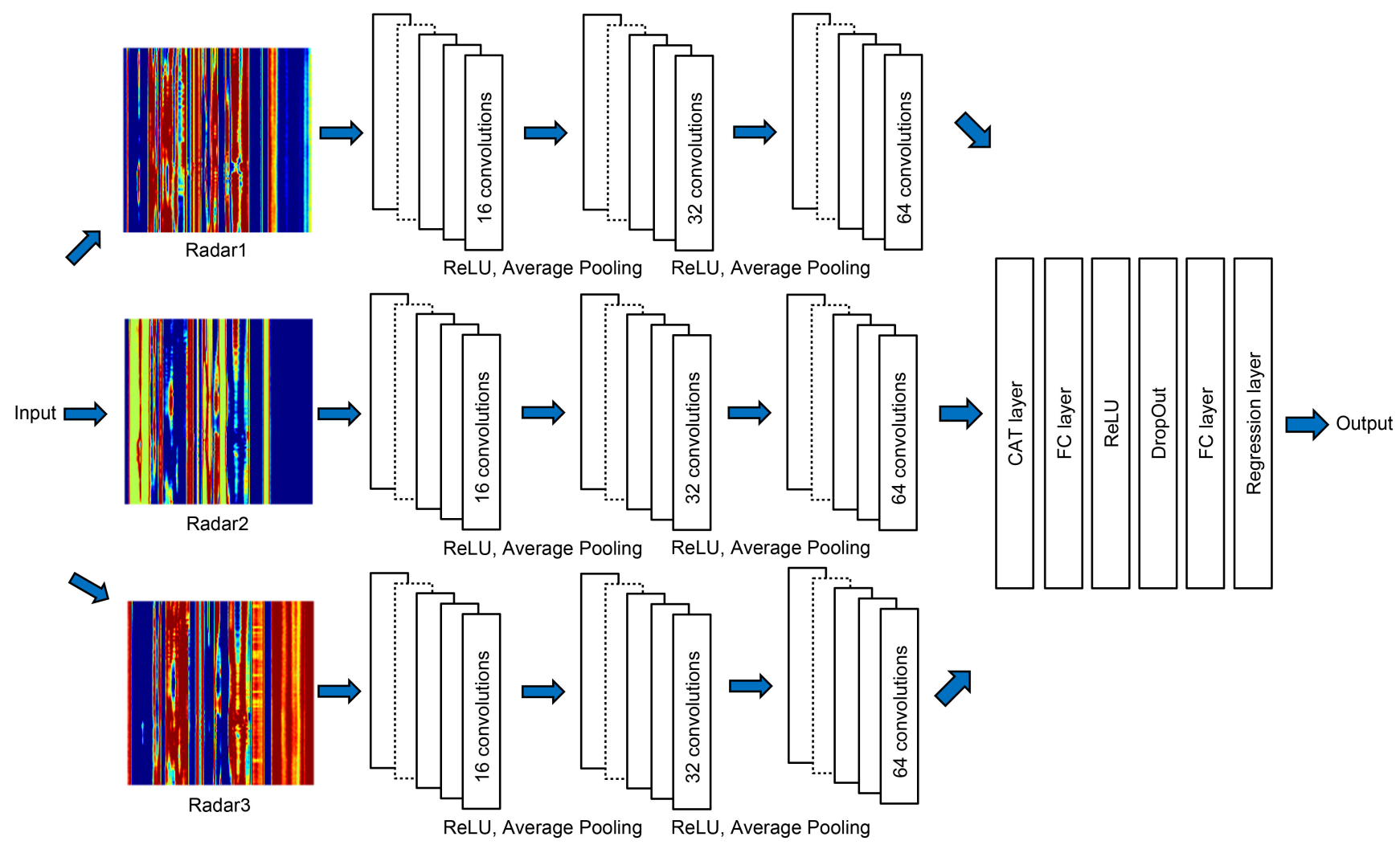

Figure 3. Architecture of the implemented convolutional neural network.

\begin{tabular}{|c|c|}
\hline Hyperparameter & Value \\
\hline Number of hidden layers in CNN & 3 \\
\hline Convolution filter size & 3 \\
\hline Learning rate & 0.001 \\
\hline Minibatch size & 8 \\
\hline Number of epochs & 60 \\
\hline
\end{tabular}

Table 1. Hyperparameter values of the proposed convolutional neural network.

\section{Results}

\section{Baseline Subject Data}

A total of 45 subjects (19 males, 26 females) were enrolled, and their characteristics are shown in Table 2. The median (interquartile range) height measured by the stadiometer was $164.00 \mathrm{~cm}$ (160.95-172.82). The median (interquartile range) weight and BMI obtained from BIA were $62.20 \mathrm{~kg}$ (55.30-74.62) and 22.80 (20.57-26.00), respectively. The median percentages of skeletal muscle mass, muscle mass, body water, and body fat were $22.70 \%$ (20.40-31.42), $39.20 \%$ (35.80-52.52), $30.60 \%$ (27.95-40.85), and $17.90 \%(15.30-22.55)$, respectively.

\section{Results of Preprocessing the Radar Signal}

Figure 4 shows the implementation of the background subtraction algorithm presented in section 3.2; all signals received from the 3 radars were subjected to the proposed algorithm, which involved removing the signals corresponding to the background detected in the raw radar signals. Even if the subject was able to stand still in the environment for 5 seconds, the signal to the 


\begin{tabular}{|c|c|}
\hline Demographics & $N=\mathbf{4 5}$ \\
\hline Age, $\mathrm{y}$ & $33(26.75-45.50)$ \\
\hline Male & $19(42.22 \%)$ \\
\hline Height, $\mathrm{cm}$ & $164.00(160.95-172.82)$ \\
\hline Weight, $\mathrm{kg}$ & $62.20(55.30-74.62)$ \\
\hline Body mass index, $\mathrm{kg} / \mathrm{m}^{2}$ & $22.80(20.57-26.00)$ \\
\hline Muscle mass, \% & $39.20(35.80-52.52)$ \\
\hline Skeletal muscle mass, \% & $22.70(20.40-31.42)$ \\
\hline Body water, \% & $30.60(27.95-40.85)$ \\
\hline Body fat, \% & $17.90(15.30-22.55)$ \\
\hline
\end{tabular}

Table 2. Baseline Subject Characteristics.

target could be acquired due to the generation of minute movements as well as those associated with breathing. Regardless of the experimental environment, the background signal can always be removed to produce a signal for training and validation so long as the installed positions of the sensors are consistent.

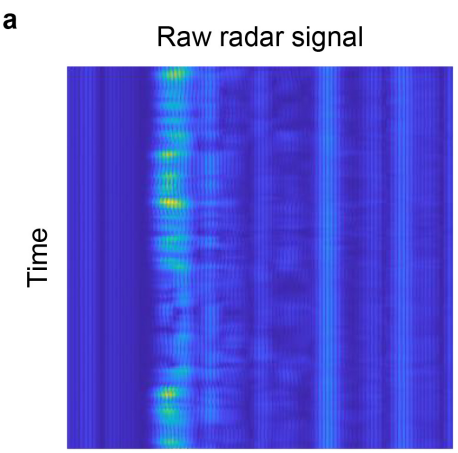

Distance

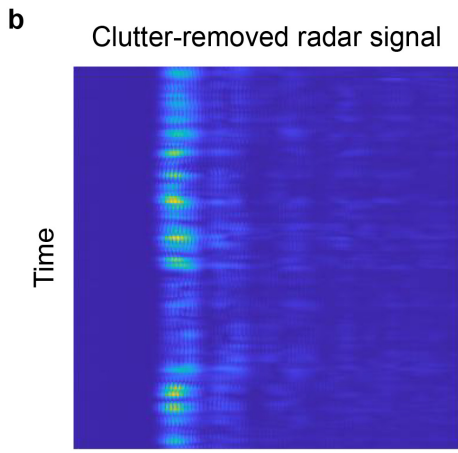

Distance

Figure 4. Background subtraction algorithm. (a) Signal before background subtraction. (b) Signal after background subtraction.

\section{Height Estimation by Signal Processing}

To estimate the height through signal processing, one data point per person was used among the collected data. Of the three radar sensors installed in the experimental environment, only the data from the sensor installed on the ceiling were used. To obtain the threshold, data were collected from the experimental environment for 5 minutes, and the height was estimated by comparing the radar signal $y_{1, n}[k]$ obtained for each participant with the threshold $T_{1}[k]$. Figure 5 is a scatter plot of the height estimated through signal processing of the preprocessed radar signal; the MAE is 1.49, and the RMSE is 3.37. The ICC value between the estimated data and the measured data is 0.92 , and the Pearson correlation coefficient $r^{2}$ is 0.85 .

\section{Estimation Results}

To assess the accuracy of the proposed CNN architecture in weight estimation, we implemented different values for the CNN depth, the number of filters for the 2D convolution layer and the number of neurons in the fully connected layer. Of a total of 360 datasets collected from 45 people, $75 \%$ were randomly selected as the training set, and the remaining $25 \%$ were selected as the validation set. In Fig. 6, different colors represent different numbers of outputs of the fully connected layer used after the CAT layer: 256 in blue, 512 in red, and 1024 in yellow. When the number of hidden layers is 2, the number of filters for the 2D convolution layer is 16 or 32, and the output of the fully connected layer is 1024, the weight estimation has the smallest RMSE, 5.31. In this work, when the number of filters used in the 2D convolution layer increased excessively, the RMSE value also increased; similarly, when the depth of the network increased, the RMSE value also increased for 256 and 512 fully connected layers. For 1024 outputs of the fully connected layer and 32, 64, and 128 2D convolution layers, the RMSE values were greater than 7, the value obtaineed for 256 and 512 fully connected layer outputs.

The networks were independently trained according to the seven measured anthropometric parameters, and all their structures had the same hyperparameter values. The diagrams in Fig. 7 illustrate the result of the proposed CNN architecture by plotting the measured and estimated values of the anthropometric parameters; all plots demonstrate positive correlations and 


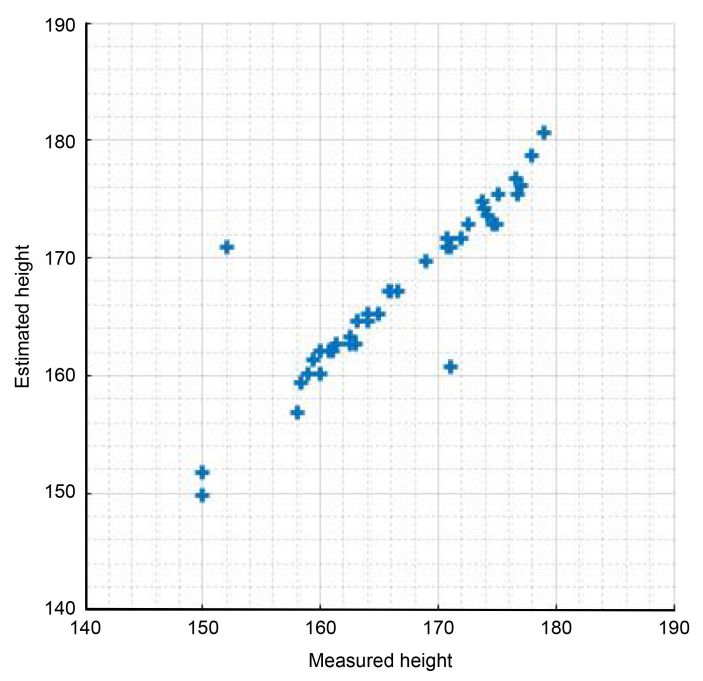

Figure 5. Comparison of estimated and measured height values.

linear distributions of the two sets of values. In Fig. 6, the error values of the heights of some of the participants were very large, but there was no such error value in the presented CNN-based results. These scatter plots show that all anthropometric parameters were sufficiently trained in the proposed structure.

\begin{tabular}{|c|c|c|c|c|}
\hline Anthropometric Parameter & RMSE & MAE & ICC & $r^{2}$ \\
\hline Height, $\mathrm{cm}$ & 2.78 & 2.36 & 0.93 & 0.87 \\
\hline Weight, $\mathrm{kg}$ & 5.31 & 4.28 & 0.94 & 0.88 \\
\hline Body mass index, $\mathrm{kg} / \mathrm{m}^{2}$ & 2.25 & 1.66 & 0.83 & 0.84 \\
\hline Skeletal muscle mass, (\%) & 1.48 & 1.16 & 0.97 & 0.95 \\
\hline Muscle mass, (\%) & 2.34 & 1.95 & 0.97 & 0.96 \\
\hline Body water, (\%) & 1.98 & 1.59 & 0.97 & 0.95 \\
\hline Body fat, (\%) & 3.36 & 2.53 & 0.90 & 0.82 \\
\hline
\end{tabular}

Table 3. Estimation Results from the CNN.

The distribution of errors for the predicted values of each anthropometric parameter is shown in Fig. 8. For each box, the mark in the center represents the median, and the bottom and top edges of the box represent the 25th and 75th percentiles, respectively. Outlier ranges indicated by dotted lines are values that are at least 1.5 times the interquartile range from the bottom or top of the box, and data displayed outside the outlier range are shown in red. The median values for the errors of all indicators are distributed within 5, and some data for body fat are beyond the range of the marked outliers. Table 3 shows the results of the network trained for each anthropometric parameter in the proposed CNN architecture. The RMSE (5.31) and MAE (4.28) for weight were higher than those for the other parameters; these values indicate an error of less than 10 percent relative to an average weight of $62.00 \mathrm{~kg}$, and the ICC value (0.96) indicates excellent agreement between the radar sensor and BIA. The ICC value of BMI (0.83) was lower than that of the other parameters; however, the absolute value of the error (RMSE 2.25 , MAE 1.66$)$ was within $10 \%$ of the average BMI $\left(22.80 \mathrm{~kg} / \mathrm{m}^{2}\right)$. The ICC values of the other parameters $(>0.90)$ and their low RMSE and MAE values also indicate excellent agreement between the radar sensor and BIA.

\section{Discussion}

In this study, our innovative, noncontact technology, IR-UWB radar, successfully measured anthropometric parameters with a high degree of accuracy comparable to that of BIA. Moreover, we demonstrated a state-of-the-art algorithm for simultaneously estimating the parameters using a machine learning scheme and a CNN architecture. The drawbacks of requiring multiple radar sensors and the time needed to acquire the signals remain a challenge that must be addressed in future work.

Anthropometric profiles are important for assessing obesity or other medical conditions that affect body weight or body shape. Obesity significantly increases the risk of cardiovascular disease, hypertension, diabetes mellitus, and many other diseases. The anthropometric profiles of infants and children need to be measured to assess their nutritional status ${ }^{2}$. Moreover, 


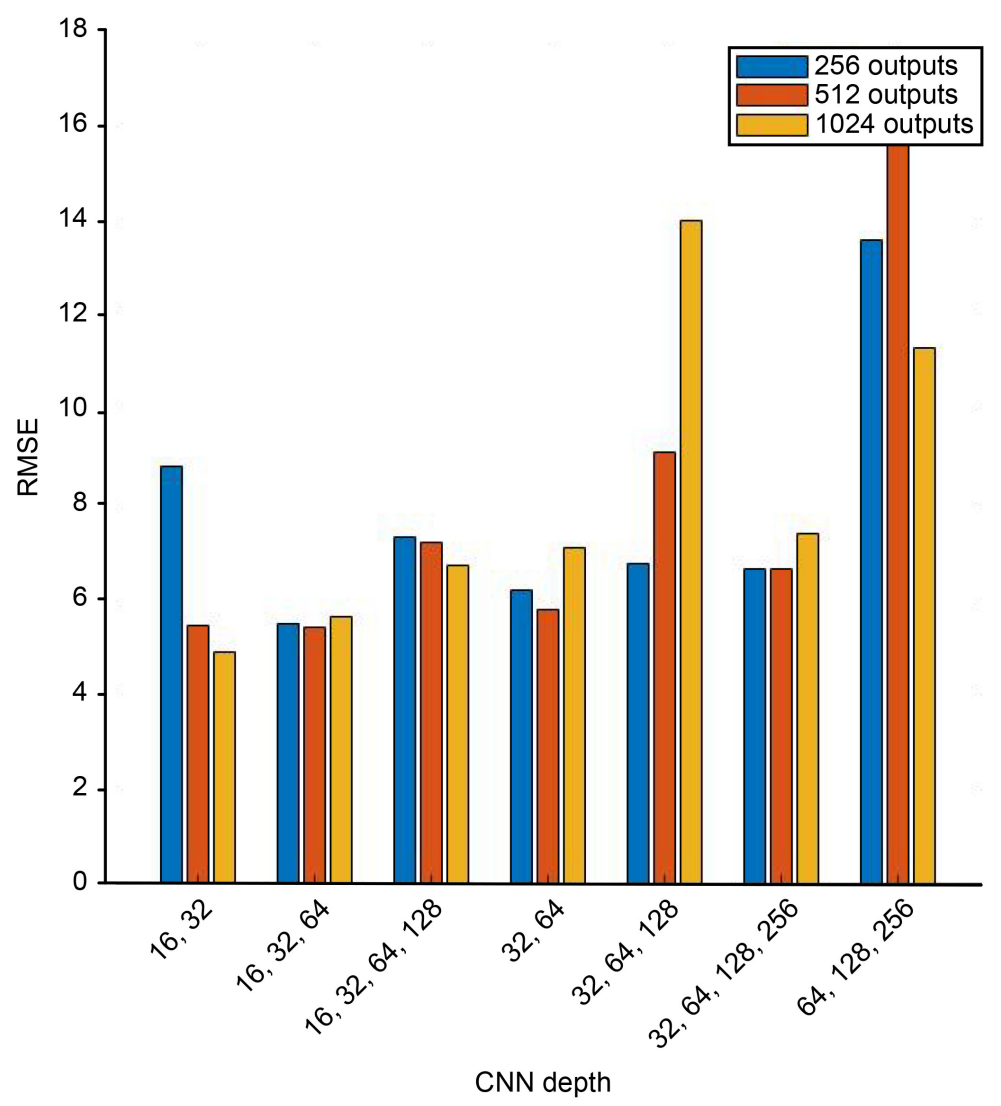

Figure 6. RMSE comparison for weight estimation.

BMI-for-age is commonly used as an index to identify the severity of obesity or malnutrition ${ }^{2}$. Additionally, anthropometric measurements can be used as a baseline for physical fitness in athletes and to measure the progress of fitness. A number of techniques have been used to assess body composition; for example, BIA is simple and easy to perform, but it requires the placement of electrodes on the body, and measurements should ideally be made with the subject at rest. Dual energy X-ray absorptiometry and computed tomography require expensive equipment and trained technicians and expose the subject to radiation $^{24-27}$.

The 3D structure light sensor (Libra3D) has been shown to be an accurate approach to weight measurement relative to other conservative techniques. There are some limitations, however, due to its reliance on optical technologies alone: (1) larger measurement errors for very muscular, obese, or very thin patients, (2) the lack of cover with a blanket, and (3) unsuitability for patients who cannot be positioned with their back on the stretcher ${ }^{4}$. Recently, a noncontact ultrasonic sensor demonstrated good results with a low average error in measuring height and weight; this device is capable of overcoming one major drawback, weight change with altitude, of traditional weight measurements using a scale, but clothes should fit tightly to the body so that the volume estimation step would give a reasonable result ${ }^{9}$. Anthropometric measurement methods from images obtained from a camera demonstrated an acceptable level of precision feasible even in unconstrained images ${ }^{10,11}$.

Noncontact monitoring technologies are playing a vital role in the COVID-19 pandemic era; as it is important to minimize contact, such technologies can lower the risk of infection when measuring anthropomorphic profiles. Individual height and weight estimation through noncontact methods or images could have considerable value in surveillance/screening for school health and emergency situations, pedestrian traffic studies for urban planning, forensic science/intrusion detection, automated garment fitting in online stores, and autonomous driving. In particular, these techniques can be used appropriately in digital healthcare markets and the COVID-19 era of non-face-to-face business and virtual environments ${ }^{10}$. IR-UWB radar is an unobtrusive, cost-effective, easily applicable, low-risk, and contactless system which, in previous studies, we suggested as a new method for measuring vital signs in a noncontact manner. We also reported the accuracy of IR-UWB radar in monitoring heart rate and respiratory rate in adults, infants and patients under nocturnal polysomnography. Through these studies, we suggested that IR-UWB radar could accurately measure infant signals despite their being of smaller amplitude and higher frequency than those of adults and is thus sufficiently clinically applicable ${ }^{6,12,28-30}$. We found that IR-UWB radar could 

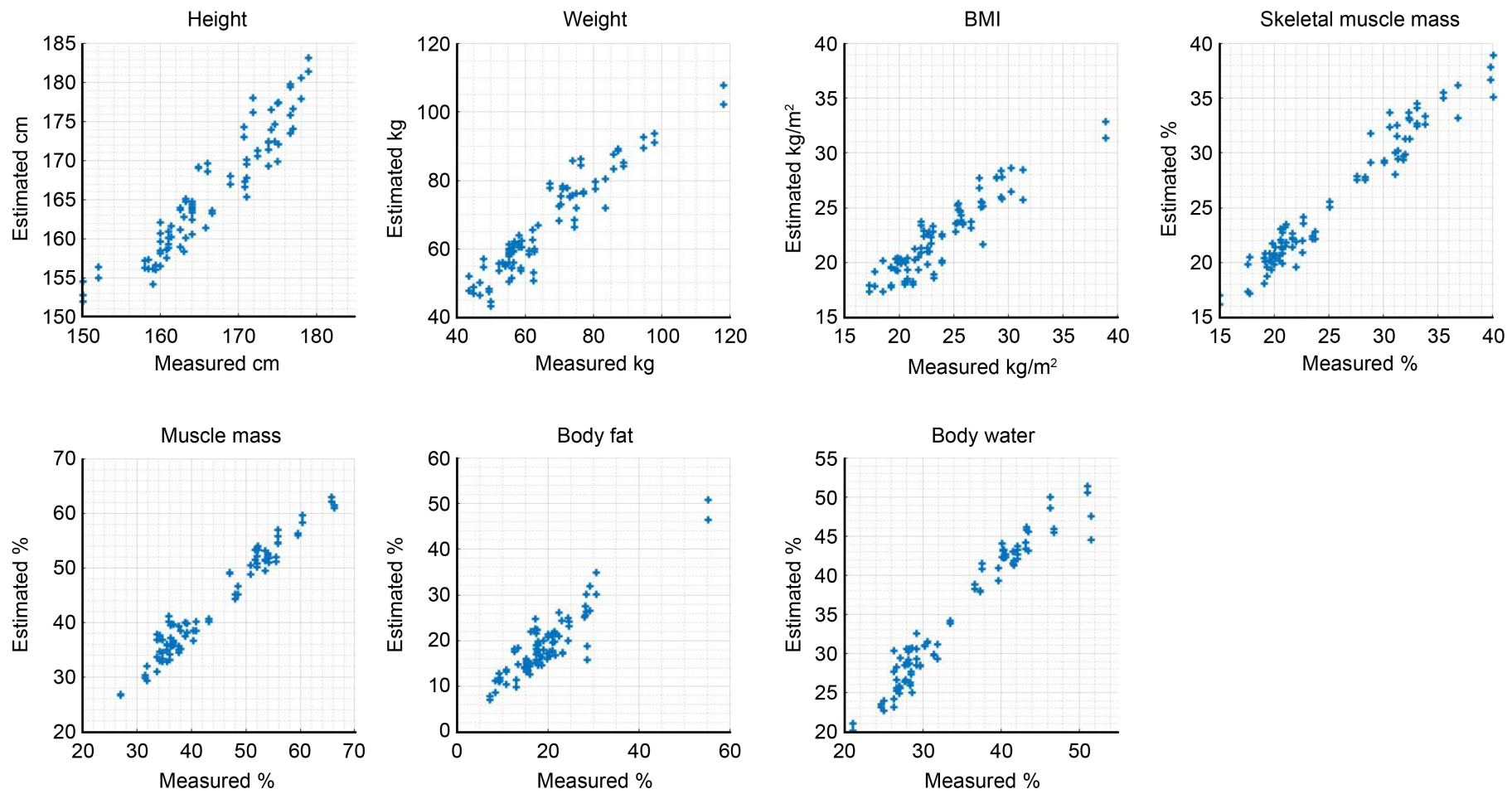

Figure 7. Comparison of estimation values and measured values of anthropometric parameters.

measure anthropometric parameters without the use of physical contact and radiation, and we validated the accuracy measured by radar for the first time.

We used three IR-UWB radar sensors for body data collection, transformed the preprocessed radar signal into an image, and then extracted the features using those images with the proposed CNN architecture. Our results showed that IR-UWB radar can accurately measure anthropometric parameters in a noncontact manner and that the accuracy of the radar system was sufficiently high, suggesting its clinical applicability in situations such as medical emergencies. Since most drug doses used in emergency situations are based on weight, especially in children, when the patient's weight cannot be measured, an accurate, rapid estimation of weight is important for administering the correct amount of medication and appropriate emergent intervention $^{31,32}$. Several methods are commonly used for weight estimation, such as visual estimation, formulas and habitus modified systems; however, these methods bear the risk of estimation errors. Even with Pediatric Advanced Weight Prediction in the Emergency Room (PAWPER) tape, the most accurate weight estimation device, the percentage of estimates within 10 percent of the actual weight is less than $80^{33-36}$. Weight estimation using radar showed better results through excellent agreement with the weight measured using BIA. The results demonstrated that IR-UWB radar can feasibly measure patient weight in medical emergency situations.

Although the proposed CNN structure is efficient with the dataset used in this study, it cannot be said to be optimal.Because the number of datasets used in this study was not large, a network with a fairly deep structure could not be used. However, the data used were not acquired at a single moment, and the input data used for the proposed CNN were time series of radar data. More optimal structures for use with larger datasets could be developed based on a transformer network, recurrent neural network or long short-term memory network ${ }^{37,38}$.

Future studies with other populations, such as adults with low fat and high physical activity or obesity, children and adolescents, are warranted to validate the methodology for more varied groups. Additionally, further study is necessary to achieve sufficient accuracy with patients in the supine position to use the IR-UWB radar as an anthropometric measuring method in clinical practice, since the supine position is the most frequently used for clinical procedures. In future work, this technique and the associated module can be implemented with the IoT for various remote health monitoring applications or emergent situations in which a scale cannot be used to measure human weight. Moreover, a real-time version of the radar system will be designed for embedded system implementation to construct a standalone hardware and software solution. 


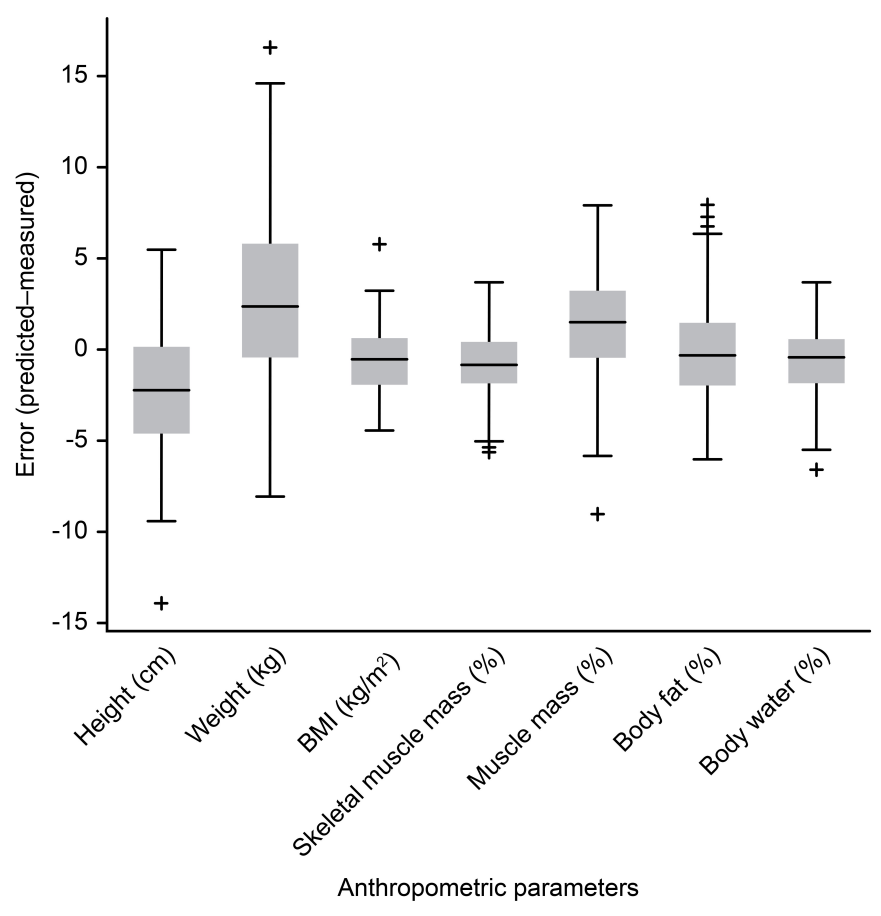

Figure 8. Box plot for estimation error.

\section{Limitations}

This study has some limitations. The main problem is the dearth of real datasets with anthropometric ground truths for individuals. Second, we used three radar sensors to acquire the signals from various angles. To improve the applicability of the proposed system in the clinical field, an algorithm must be developed that possesses sufficient accuracy with a smaller number of radar sensors.

\section{Conclusions}

For the first time, we demonstrated that an innovative, noncontact, novel, effective method, IR-UWB radar technology integrated with a machine learning algorithm, can measure anthropometric profiles successfully with substantial agreement with BIA in adults. Further studies with younger age groups and better algorithms are warranted before the proposes system can be widely used in clinical practice with the robustness of radar.

\section{References}

1. de Onis, M. 4.1 the who child growth standards. Pediatr. Nutr. Pract. 113, 278-294 (2015).

2. Guideline: assessing and managing children at primary health-care facilities to prevent overweight and obesity in the context of the double burden of malnutrition. Updates for the Integrated Management of Childhood Illness (IMCI). Geneva: World Health Organization (2017).

3. McDonald, C. M. et al. The effect of multiple anthropometric deficits on child mortality: Meta-analysis of individual data in 10 prospective studies from developing countries. Am. J. Clin. Nutr. 97, 896-901, DOI: 10.3945/ajcn.112.047639 (2013). https://academic.oup.com/ajen/article-pdf/97/4/896/23819186/896.pdf.

4. Pfitzner, C. et al. Libra3d: Body weight estimation for emergency patients in clinical environments with a $3 \mathrm{~d}$ structured light sensor. In 2015 IEEE International Conference on Robotics and Automation (ICRA), 2888-2893 (IEEE, 2015).

5. Kevat, A. C., Bullen, D. V. R., Davis, P. G. \& Kamlin, C. O. F. A systematic review of novel technology for monitoring infant and newborn heart rate. Acta Paediatr. 106, 710-720, DOI: https://doi.org/10.1111/apa.13786 (2017). https: //onlinelibrary.wiley.com/doi/pdf/10.1111/apa.13786.

6. Lee, W. H. et al. Feasibility of non-contact cardiorespiratory monitoring using impulse-radio ultra-wideband radar in the neonatal intensive care unit. PLoS One 15, e0243939 (2020). 
7. Xu, H., Yu, Y., Zhou, Y., Li, Y. \& Du, S. Measuring accurate body parameters of dressed humans with large-scale motion using a Kinect sensor. Sensors 13, 11362-11384 (2013).

8. Huang, L. et al. Non-contact body measurement for qinchuan cattle with LiDAR sensor. Sensors 18, 3014 (2018).

9. Ly, M. H., Khang, N. M., Nhi, T. T., Dang, T. T. \& Dinh, A. A non-contact human body height and weight measurement approach using ultrasonic sensor. In International Conference on the Development of Biomedical Engineering in Vietnam, 31-37 (Springer, 2018).

10. Altinigne, C. Y., Thanou, D. \& Achanta, R. Height and weight estimation from unconstrained images. In ICASSP 2020-2020 IEEE International Conference on Acoustics, Speech and Signal Processing (ICASSP), 2298-2302 (IEEE, 2020).

11. Burini, S. et al. Non-contact anthropometric measurements in newborn patients. In 2020 IEEE International Symposium on Medical Measurements and Applications (MeMeA), 1-5 (IEEE, 2020).

12. Park, J.-Y. et al. Preclinical evaluation of noncontact vital signs monitoring using real-time IR-UWB radar and factors affecting its accuracy. Sci. Rep. 11, 1-12 (2021).

13. Lee, W. H. et al. Quantified assessment of hyperactivity in adhd youth using IR-UWB radar. Sci. Rep. 11, 1-10 (2021).

14. Yim, D. et al. Quantified activity measurement for medical use in movement disorders through IR-UWB radar sensor. Sensors 19, 688 (2019).

15. Kim, J. D. et al. Non-contact respiration monitoring using impulse radio ultrawideband radar in neonates. Royal Soc. Open Sci. 6, 190149 (2019).

16. Wang, D., Yoo, S. \& Cho, S. H. Experimental comparison of IR-UWB radar and FMCW radar for vital signs. Sensors 20, 6695 (2020).

17. Wang, H. et al. Estimation of prevalence of sarcopenia by using a new bioelectrical impedance analysis in Chinese community-dwelling elderly people. BMC Geriatr. 16, 1-9 (2016).

18. Choi, J. W., Yim, D. H. \& Cho, S. H. People counting based on an IR-UWB radar sensor. IEEE Sens. J. 17, 5717-5727 (2017).

19. Rakibe, R. S. \& Patil, B. D. Background subtraction algorithm based human motion detection. Int. J. Sci. Res. Publi. 3, 2250-3153 (2013).

20. Maali, A. et al. Ca-CFAR threshold selection for IR-UWB TOA estimation. In International Workshop on Systems, Signal Processing and Their Applications, WOSSPA, 279-282 (IEEE, 2011).

21. Park, J.-Y. et al. Preclinical evaluation of a noncontact simultaneous monitoring method for respiration and carotid pulsation using impulse-radio ultra-wideband radar. Sci. Rep. 9, 1-12 (2019).

22. Joze, H. R. V., Shaban, A., Iuzzolino, M. L. \& Koishida, K. MMTM: Multimodal transfer module for CNN fusion. In Proceedings of the IEEE/CVF Conference on Computer Vision and Pattern Recognition, 13289-13299 (2020).

23. Gadzicki, K., Khamsehashari, R. \& Zetzsche, C. Early vs late fusion in multimodal convolutional neural networks. In 2020 IEEE 23rd International Conference on Information Fusion (FUSION), 1-6 (IEEE, 2020).

24. Tewari, N., Awad, S., Macdonald, I. A. \& Lobo, D. N. A comparison of three methods to assess body composition. Nutrition 47, 1-5 (2018).

25. Lee, S. Y. \& Gallagher, D. Assessment methods in human body composition. Curr. Opin. Clin. Nutr. Metab. Care 11, 566 (2008).

26. Lyons-Reid, J., Ward, L. C., Kenealy, T. \& Cutfield, W. Bioelectrical impedance analysisâ€”an easy tool for quantifying body composition in infancy? Nutrients 12, 920 (2020).

27. Brantlov, S., Ward, L. C., Jødal, L., Rittig, S. \& Lange, A. Critical factors and their impact on bioelectrical impedance analysis in children: A review. J. Med. Eng. Technol. 41, 22-35 (2017).

28. Lee, Y. et al. A novel non-contact heart rate monitor using impulse-radio ultra-wideband (IR-UWB) radar technology. Sci. Rep. 8, 1-10 (2018).

29. Kang, S. et al. Validation of noncontact cardiorespiratory monitoring using impulse-radio ultra-wideband radar against nocturnal polysomnography. Sleep Breath. 24, 841-848 (2020).

30. Lee, W. H. et al. Non-contact sleep/wake monitoring using impulse-radio ultrawideband radar in neonates. Front. Pediatr. 9, 1471, DOI: 10.3389/fped.2021.782623 (2021). 
31. Meguerdichian, M. J. \& Clapper, T. C. The broselow tape as an effective medication dosing instrument: A review of the literature. J. Pediatr. Nurs. 27, 416-420 (2012).

32. Luten, R. \& Zaritsky, A. The sophistication of simplicity... optimizing emergency dosing. Acad. Emerg. Med. 15, 461-465 (2008).

33. Cattermole, G. N. \& Manirafasha, A. Accuracy of weight estimation methods in adults, adolescents and children: A prospective study. Emerg. Med. J. 38, 718-723 (2021).

34. Lin, B. W., Yoshida, D., Quinn, J. \& Strehlow, M. A better way to estimate adult patients' weights. Am. J. Emerg. Med. 27, 1060-1064 (2009).

35. Wells, M., Goldstein, L. N. \& Bentley, A. The accuracy of emergency weight estimation systems in childrenâ€"a systematic review and meta-analysis. Int. J. Emerg. Med. 10, 1-43 (2017).

36. Wells, M. \& Goldstein, L. N. Optimising emergency weight estimation in underweight and obese children: the accuracy of estimations of total body weight and ideal body weight. Eur. J. Emerg. Med. 26, 301-307 (2019).

37. Bai, J. et al. Radar transformer: An object classification network based on 4d mmw imaging radar. Sensors 21, 3854 (2021).

38. Wang, L., Giebenhain, S., Anklam, C. \& Goldluecke, B. Radar ghost target detection via multimodal transformers. IEEE Robot. Autom. Lett. 6, 7758-7765 (2021).

\section{Funding}

This research was supported by the Bio and Medical Technology Development Program (Next Generation Biotechnology) through the National Research Foundation of Korea (NRF) funded by the Ministry of Science, ICT and Future Planning (NRF-2017M3A9E2064735) and the research fund of Hanyang University MEB (Global Center For Developmental Disorders, HY-201900000003070).

\section{Author contributions statement}

Conceptualization, Y.H.L., Seok.H.C., H.K.P. and Sung.H.C.;methodology, W.H.L. and D.W.; software, W.H.L. and D.W.; validation, W.H.L. and D.W.; formal analysis, W.H.L. and D.W.; investigation, W.H.L. and D.W.; resources, Sung.H.C.; data curation, W.H.L. and D.W.; writing—original draft preparation, J.K., W.H.L, S.H.K. and H.K.P.; writing—review and editing, J.K., W.H.L., S.H.K. and J.Y.N.; visualization, J.K., W.H.L., S.H.K. and J.Y.N.; supervision, H.K.P. and Sung.H.C.; project administration, W.H.L.; funding acquisition, H.K.P. and Sung.H.C. All authors reviewed the manuscript. 